\title{
Demonstration of an absolute Rayleigh scattering spectral calibration on the W7-X Thomson scattering system
}

\author{
E.R. Scott, ${ }^{a}, 1$ M.N.A. Beurskens, ${ }^{a}$ S.A. Bozhenkov, ${ }^{a}$ G. Fuchert, ${ }^{a}$ M. Hirsch, ${ }^{a}$ P. Nelde ${ }^{b}$ E. \\ Pasch, ${ }^{a}$ R.C. Wolf, ${ }^{a, b}$ the W7-X Team \\ ${ }^{a}$ Max-Planck-Institut für Plasmaphysik, D-17491 Greifswald, Germany \\ ${ }^{b}$ Technische Universität Berlin, D-10623 Berlin, Germany \\ E-mail: evan.scott@ipp.mpg.de
}

\begin{abstract}
Aвstract: A novel in-situ calibration method for Thomson scattering (TS) diagnostics has been demonstrated on the Wendelstein 7-X stellarator (W7-X). Utilizing a laser-pumped optical parametric oscillator (OPO), Rayleigh scattering measurements were performed in-situ in argon, nitrogen, and air at pressures from 0 to 1024 mbar and wavelengths between 730 and $1070 \mathrm{~nm}$. As the OPO follows the same beam path as the TS lasers, the scattering angle and solid angle of collection can be made identical to the TS geometry; therefore, the calibration covers all optical components within the TS system, including the first vacuum window, and can, in principle, be used to directly calibrate each filter channel for absolute plasma density. Despite high background stray light levels, the Rayleigh scattered signal was successfully isolated from the stray light, and filter curves for multiple polychromators were resolved. The complete calibration system, planned for future W7-X operational campaigns, will aim to further reduce stray light, thereby decreasing the required gas pressure and allowing in-situ calibration measurements on service days. This is planned in part by better matching the OPO beam alignment, size, and divergence to the installed Nd:YAG TS lasers. In addition, the OPO bandwidth will be improved by using an injection seeder to increase filter edge resolution.
\end{abstract}

KeYwords: Detector alignment and calibration methods (lasers, sources, particle beams); Plasma diagnostics - interferometry, spectroscopy, and imaging; Lasers

\footnotetext{
${ }^{1}$ Corresponding author.
} 


\section{Introduction}

In magnetic confinement fusion devices, Thomson scattering (TS) diagnostics are widely used to determine electron temperature and density profiles by measuring the elastic scattering of light off of electrons gyrating about magnetic field lines used to confine the plasma [1-10]. The distribution of electron temperatures results in a Doppler-shifted scattered spectrum. Therefore, by measuring the scattered spectrum, the original electron temperature can be reproduced; the absolute signal intensity, however, is directly proportional to the number of scattering events, and therefore the electron density.

The simultaneous measurement of electron temperature and density by TS requires careful diagnostic calibration. Most TS systems perform two distinct calibrations to accurately determine temperature and density profiles: a spectral calibration to measure the spectral transmission of the system, including the polychromator bandpass filters; and an absolute calibration to determine the signal intensity per number of scattering events.

The Wendelstein 7-X optimized stellarator (W7-X) uses a full-profile TS system capable of measuring temperature and density profiles across the entire plasma [10]. During the most recent operational period in late 2018, signals from 42 scattering volumes were measured using 20 polychromators, with plans to increase the number of scattering volumes to 95 for future campaigns. An absolute calibration of the TS system was performed via Raman scattering, and a spectral calibration via a supercontinuum laser, monochromator, and diffuse reflecting plate [10].

The Raman scattering density calibration, performed in nitrogen at pressures varying from 100 to 0 mbar, uses the same setup and Nd:YAG lasers as are used during Thomson scattering measurements, yielding the same geometric factors for the calibration and temperature/density measurements [10-12]. Additionally, the calibration is unaffected by stray light. It is, however, only capable of absolutely calibrating one of five polychromator channels consistently; a second channel can be calibrated, but signal levels are significantly lower, resulting in larger uncertainties. The absolute calibration is used in conjunction with a spectral calibration to scale the remaining channels [10].

The diffuse-plate spectral calibration method is performed by illuminating a diffuse reflecting plate with one wavelength selected by a monochromator. The optics are withdrawn from the vacuum vessel, and the diffuse reflecting plate is set in front of the optics. This spectral calibration method has a bandwidth determined only by the monochromator and by the required signal levels. Additionally, a large number of points per wavelength are recorded due to the near $30 \mathrm{kHz}$ repetition rate of the supercontinuum laser, resulting in low statistical noise over a relatively short acquisition period.

However, the diffuse reflection calibration does not measure the first vacuum window, nor does it measure from the focal point of the collection optics; the diffuse reflecting plate is placed approximately $5 \mathrm{~cm}$ from the collection optics rather than the normal $1.3 \mathrm{~m}$ from the optics to the scattering volume in the plasma [10]. This effectively overfills the scattering volumes, potentially making the calibration less representative of what is seen during Thomson scattering measurements.

A calibration method for determining both the spectral and absolute sensitivity of a TS system via Rayleigh scattering has been proposed previously [13], with laboratory measurements yielding promising results [14]. In this method, light from a wavelength-tunable source is directed along the 
same beam path normally used by the TS laser during plasma operation. When the plasma vessel is filled with a gas, i.e. argon or nitrogen, Rayleigh scattering occurs along the beam path. The scattered light is then collected and measured using the standard TS system's optics and detectors. The wavelength-tunable source scans through all wavelengths relevant to the TS system, giving a full spectral sensitivity that includes all optical components, and has the potential to use the same beam and scattering geometry as the standard TS system through proper beam shaping and positioning. With a well-collimated source, such as a Nd:YAG-based optical parametric oscillator (OPO), the number of scattering events at a given wavelength can be determined, allowing for absolute density calibration in addition to spectral calibration if the beam profile of the calibration laser at the vessel entrance and exit match those of the Nd:YAG lasers used during plasma measurements.

However, unlike the diffuse reflection calibration, the Rayleigh scattering calibration results are highly dependent on stray light levels. In the presence of significant stray light, high pressures or very long measurements are needed to see Rayleigh scattering signals comparable to those of the stray light. Additionally, the OPO repetition rate of $10 \mathrm{~Hz}$ is significantly lower than the $30 \mathrm{kHz}$ supercontinuum laser, resulting in higher statistical noise even when data is collected over a longer period.

In this work, the viability of the Rayleigh scattering spectral calibration is shown via proofof-principle measurements performed at W7-X. These measurements were performed in argon, nitrogen, and air using a Nd:YAG-based OPO. Despite high stray light levels, filter transmission curves for the majority of polychromators were obtained for measurements in air, with some channels resolved in argon. Differences to the previously used spectral calibration technique are observed. Density calibration factors were also determined for all wavelengths within the range of the polychromators; however, these factors do not agree with those determined via Raman scattering, as will be discussed in section 3.2.

\section{Experimental setup}

After the conclusion of the late 2018 W7-X operational campaign, proof-of-principle Rayleigh scattering calibration measurements were performed in argon, nitrogen, and air. The OPO-laser was installed on the Thomson scattering optics' mechanical support structure within the W7-X torus hall and directed through the vacuum vessel using custom broadband mirrors developed by Laseroptik $\mathrm{GmbH}$. The correct beam polarization was achieved using three separate mirrors.

For beam focusing and shaping, two different methods were used. During argon and nitrogen measurements, an uncoated 2399 mm radius C7980 quartz glass lens from Laser Components GmbH was used. During measurements in air, a Galilean telescope consisting of a $50 \mathrm{~mm}$ focal length bi-concave quartz lens and a $100 \mathrm{~mm}$ focal length bi-convex quartz lens were used. Additionally, an iris aperture was included during measurements in air to further reduce the OPO beam energy.

The OPO-laser pulse energy was measured after exiting the vacuum vessel using a pyroelectric energy monitor (PEM 45 USB from Sensor-und Lasertechnik) capable of handling up to $80 \mathrm{~mJ} / \mathrm{cm}^{2}$ with a flat spectral response between 700 and $1100 \mathrm{~nm}$. During argon and nitrogen measurements, the OPO beam was directed to the PEM by another broadband mirror to allow for beam position control and monitoring via cameras behind the entrance and exit mirrors. However, due to the suspicion that the mirror coating could be faulty, the beam was dumped directly onto the PEM 
during measurements in air to ensure an accurate pulse energy measurement. No noticeable increase in stray light levels was observed.

The system setup is shown in figure 1 .

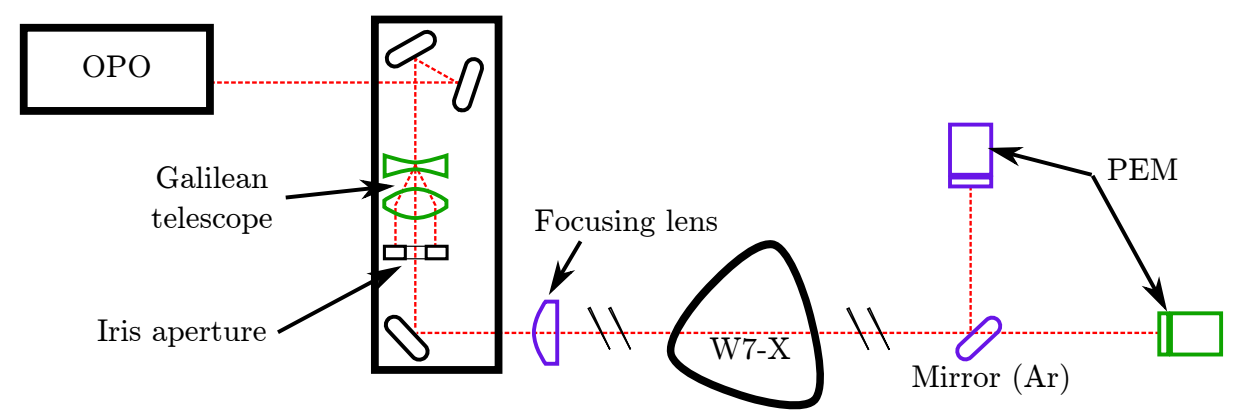

Figure 1. OPO-laser setup used during Rayleigh scattering measurements. For measurements in argon and nitrogen, a single $2399 \mathrm{~mm}$ plano-convex quartz glass lens focused the beam, and a mirror after the vacuum vessel directed the exit beam toward the PEM (purple). For measurements in air, a Galilean telescope with one $50 \mathrm{~mm}$ focal length bi-concave lens and one $100 \mathrm{~mm}$ focal length bi-convex lens replaced the plano-convex lens, and the beam was dumped directly onto the PEM (green). Components shown in black are common to all measurements.

A wavelength scan from 730 to $1070 \mathrm{~nm}$ was performed at each pressure in $1 \mathrm{~nm}$ increments. For greater resolution when measuring the Raman-calibrated spectral channels, an additional wavelength scan from 1040 to $1060 \mathrm{~nm}$ was performed in $0.1 \mathrm{~nm}$ steps. A total of 15 OPO pulses were measured at each wavelength step in argon and nitrogen. To improve the signal-to-noise, 30 OPO pulses were measured at each wavelength step during measurements in air. Note that, because the OPO linewidth varies from $0.23 \mathrm{~nm}$ at $680 \mathrm{~nm}$ to $0.73 \mathrm{~nm}$ at $1100 \mathrm{~nm}$, the step size of the high resolution scan is smaller than the OPO linewidth.

For measurements in argon, the vessel was first brought to 100 mbar and pumped-down to 50 and 0 mbar. The vessel was then filled with nitrogen for an additional measurement at 100 mbar. Note that the maximum pressure differential between the W7-X cryostat and vacuum vessel is 100 mbar, thus limiting the pressure for initial measurements. This also constitutes a basic limitation for measurements during a campaign. To increase signal-to-noise, measurements were repeated in air at $0,350,500,750$, and 1013 mbar while venting the vacuum vessel to atmospheric pressure, thereby removing the low pressure limitation.

\section{Results}

The absolute calibration factor at each wavelength is determined using equation (3.1):

$$
\left(\int s d t\right)_{i} \cdot E_{0}(\lambda)^{-1}=I_{s t r}(\lambda)+n_{g a s} \cdot \frac{\lambda}{h c} \cdot \frac{d \sigma(\lambda)}{d \Omega} \cdot \delta L \delta \Omega g_{i}(\lambda)
$$

where $\left(\int s d t\right)_{i}$ is the averaged integrated avalanche photodiode signal at a given wavelength for spectral channel $i, E_{0}(\lambda)$ is the averaged OPO pulse energy at a given wavelength, $I_{s t r}(\lambda)$ is the intensity of stray light, $n_{\text {gas }}$ is the particle density of the scattering medium, $d \sigma(\lambda) / d \Omega$ is 
the differential Rayleigh scattering cross section at wavelength $\lambda$, and the product $\delta L \delta \Omega g_{i}(\lambda)$ is the absolute calibration factor, consisting of the scattering volume length, the scattering volume observation solid angle, and the absolute sensitivity of spectral channel $i$ at wavelength $\lambda$.

The relative sensitivity is given by $g_{i}(\lambda) / g_{0}\left(\lambda_{\text {ref }}\right)$, and can be determined by normalizing the absolute calibration factor of channel $i$ at a wavelength $\lambda$ to the absolute calibration factor of a selected channel at an arbitrary reference wavelength $\lambda_{r e f}$. This relative sensitivity is analogous to that determined using the diffuse scattering plate spectral calibration.

The differential Rayleigh scattering cross-sections in argon and air are determined following the method by LeBlanc in ref. [12]. The extrapolated curves from previous measurements in argon at 693 and $532 \mathrm{~nm}[15,16]$ and in standard air at $1000 \mathrm{~nm}$ [17] are modeled using the $\lambda^{-4}$ dependence of Rayleigh scattering on wavelength. The total cross-section is converted to the differential crosssection using eq. (50) in ref. [18]. For argon, the value of the differential cross-section is averaged over both measurements for each wavelength.

To normalize the scattered signals to the beam energy, the scattered signals are first averaged. This averaged signal is then normalized to the average energy measurement for all pulses at a given wavelength and pressure. For a linearly correlated set of signal and energy measurements, this method is accurate. If the signals are not linearly correlated, however, this method may yield incorrect results. For this work, it is assumed that the scattered signals and the energy measurements are, in fact, linearly correlated, and the normalized average signal is used here as a means to reduce the effects of the background noise level on the integrals of the scattered signals.

Unless otherwise mentioned, the data presented here is for volume 1025, the 25th scattering volume of the $\mathrm{N}$-port collection optics [10]; this corresponds to the first of two pulses detected using polychromator 11 .

\subsection{Argon measurements}

The measured laser energy at 100, 50, and 0 mbar for measurements in argon is given in figure 2 . The OPO pulse-to-pulse energy variation is fairly large, as is the energy dependence on wavelength; this is consistent with previous findings [14]. For the 100 mbar case, signal saturation around 840 $\mathrm{nm}$ results in the energies shown being uncharacteristically low as saturated signals are removed from the data analysis.

Figure 3 shows the raw pulse shape for a scattering volume at multiple pressures. It is clear from the large signal at 0 mbar that stray light constitutes a large portion of the detected signal. The increase in signal level with pressure is the result of Rayleigh scattering. Several scattering volumes also exhibit a double-peak rather than a single peak due to the increased time for the stray light to reach the collection optics as opposed to the Rayleigh-scattered light (see section 4).

The absolute signal intensity at a given wavelength is determined by fitting the data linearly, as the magnitude of Rayleigh scattering varies linearly with pressure. By rearranging eq. (3.1), the slope of this linear fit gives the absolute calibration factor of a given channel at that wavelength. This is shown in figure 4, where the channel and the selected wavelength are given in the figure legend. The data set showing the sensitivity of channel 1 at $1054 \mathrm{~nm}$ demonstrates that, outside of the bandpass of the filter, the spectral channel is no longer sensitive and has no response with increasing particle density. 


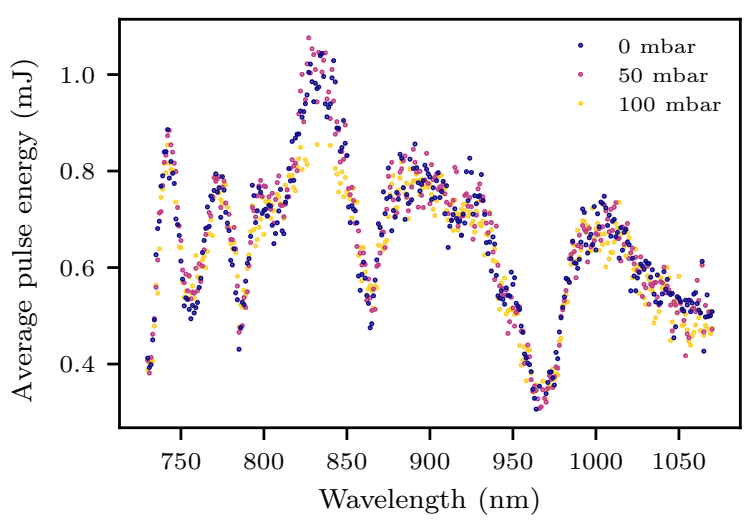

Figure 2. Pulse energy measured with a PEM after exiting the vacuum vessel. Missing data points around $830 \mathrm{~nm}$ during $100 \mathrm{mbar}$ measurements are due to removal of energy data when polychromator diodes saturate.

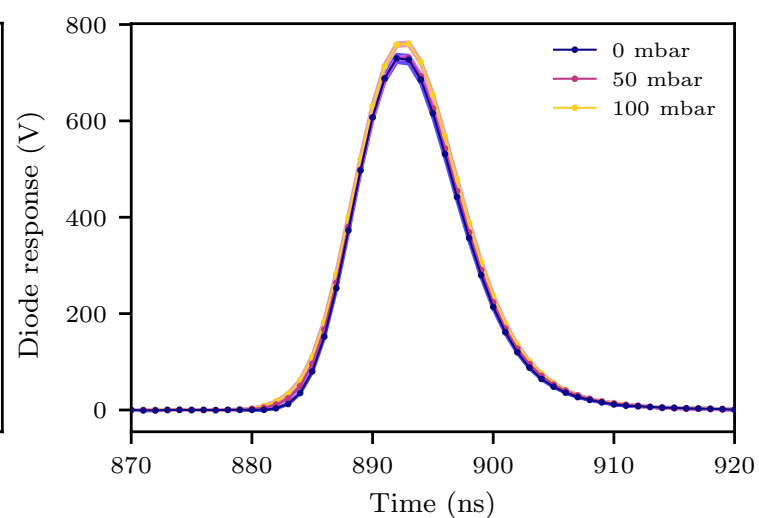

Figure 3. Averaged signal peaks over 15 OPO pulses measured at $811 \mathrm{~nm}$. Note that the 0 mbar measurement representing the stray light level constitutes the vast majority of the measured signal level, though some effect from Rayleigh scattering is visible at 50 and 100 mbar. The shaded area represents the 2-sigma standard error of the mean.

Using the slope of the fitted line for each wavelength of interest, a curve of absolute calibration factors for channel 1 is shown in figure 5. Additionally, by normalizing to some arbitrary value, a spectrally resolved relative calibration useable with a different absolute calibration method is obtained.

Though the diffuse plate spectral calibration data is usually normalized to $1054 \mathrm{~nm}$ in spectral channel 5, because signal-to-noise during argon measurements was too low to resolve longerwavelength spectral channels, values for the relative spectral calibration are normalized to $773 \mathrm{~nm}$ due to the lower statistical noise at this wavelength, and only channel 1 is shown.

To improve future calibration measurements, both the statistical error and stray light levels should be lowered. While the statistical error can be easily improved by increasing the number of measurements per wavelength, improving stray light levels requires installation of additional stray light absorbers and better OPO beam shaping to reduce divergence.

\subsection{Air measurements}

The measured laser energy for measurements in air at 0, 350, 500, 750, and 1013 mbar are shown in figure 6 . Though the energy measurements at 0 mbar are systematically lower than those at other pressures, the overall shape of the measurements agree with one another, as well as those presented during measurements in argon. Examples of the measured signal peaks at different pressures are given in figure 7.

Following the same procedure outlined in section 3.1, a linear fit is produced at every wavelength within the scan range for all 5 spectral channels (figure 8). Because of the higher gas pressure, signal-to-noise is greatly increased during measurements in air. 


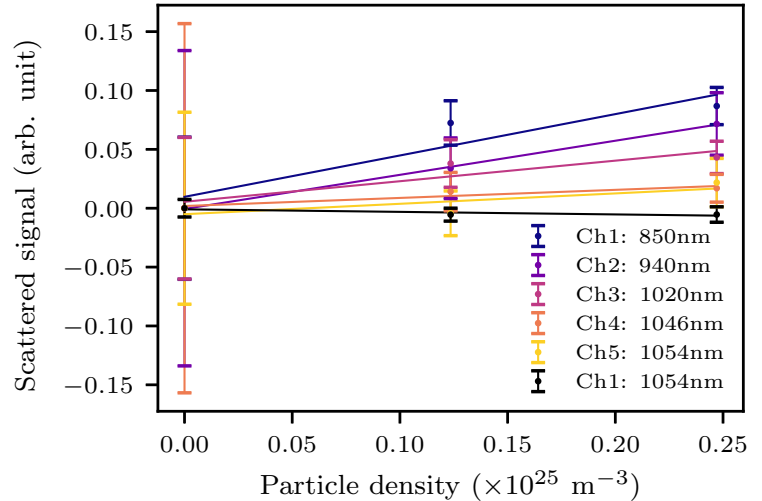

Figure 4. Linear fits of the diode response of a particular channel versus particle density at a given wavelength in argon. The positive slope illustrates the linearly increasing signal level with increasing pressure. The line for channel 1 at $1054 \mathrm{~nm}$ demonstrates that the channel is not sensitive at that wavelength, and therefore an increase in pressure does not affect the diode response. The slope of the linear fit gives the value of the absolute calibration factor or, when normalized to an arbitrary wavelength, the relative sensitivity of the spectral channel at the wavelengths indicated in the legend. Error bars denote the 2-sigma standard error of the mean.

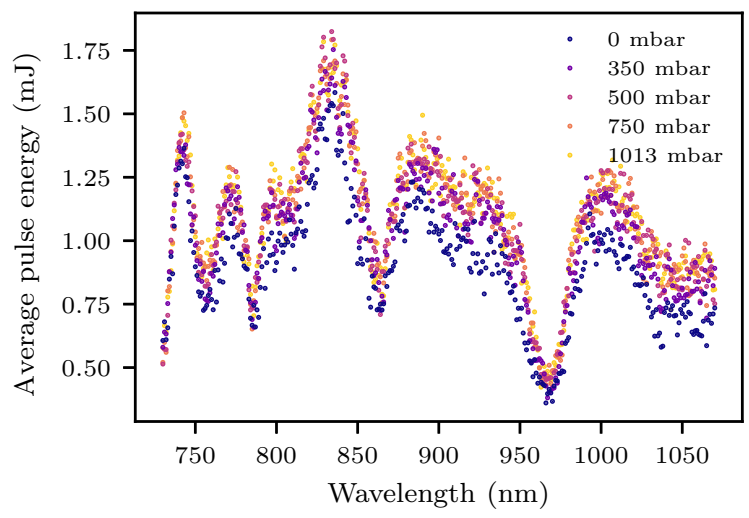

Figure 6. Pulse energy measured with a PEM after exiting the vacuum vessel during measurements in air. The signal shape closes matches that of figure 2 despite the removal of a mirror located in front of the PEM (figure 1).

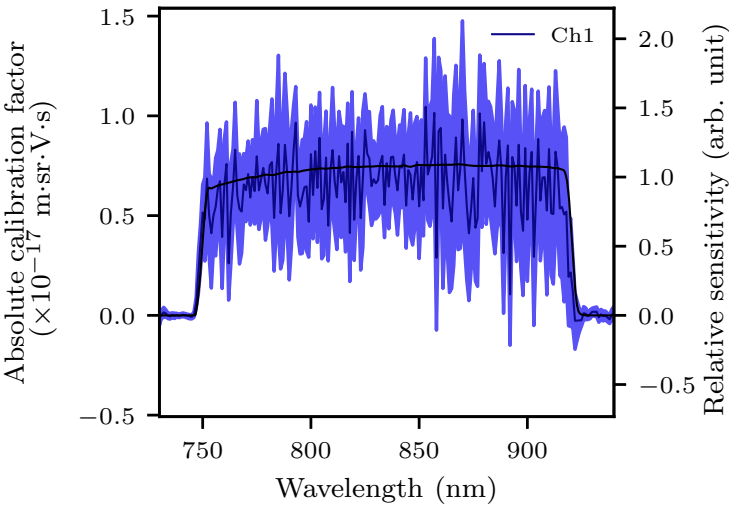

Figure 5. Absolute calibration factor and relative calibration factor normalized to $773 \mathrm{~nm}$ as determined by Rayleigh scattering in argon for spectral channel 1. The shaded area represents the 2 -sigma standard error of the mean. The diffuseplate spectral calibration is shown in black.

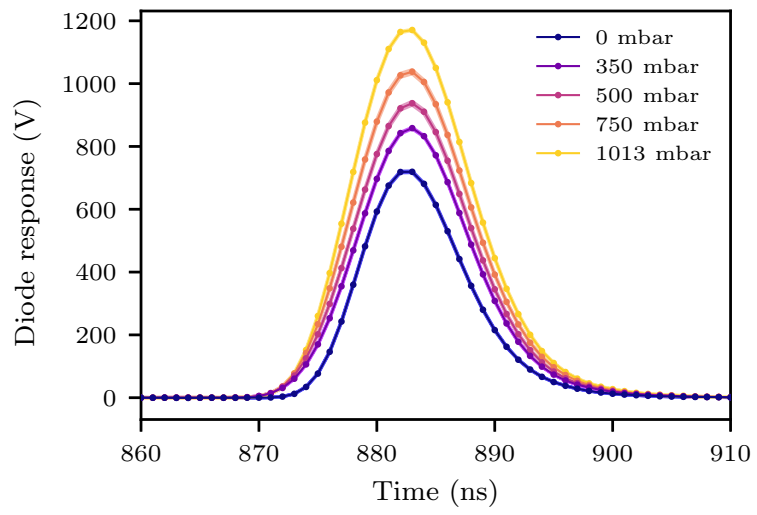

Figure 7. Averaged signal peaks over $30 \mathrm{OPO}$ pulses measured at $811 \mathrm{~nm}$. Unlike figure 3, the stray light level constitutes only half of the total signal level at maximum pressure (1013 mbar). The shaded area represents the 2-sigma standard error of the mean. 
The resultant spectral curves are shown in figure 9 , with the relativity sensitivity values resulting from normalization to $1054 \mathrm{~nm}$ as is performed for the diffuse plate relative spectral calibration. Note that, although only one polychromator is shown here, these measurements were successful for more than half of the system's polychromators despite the short data collection period imposed by time constraints. The unsuccessful reproduction of some polychromators' spectral channels is caused by several factors, including different gain levels in the avalanche photodiodes in the polychromators causing some channels to saturate before others; potential laser misalignment through the scattering volumes; and low pulse energy levels within scattering volumes closer to the beam exit window due to divergence and aberration.

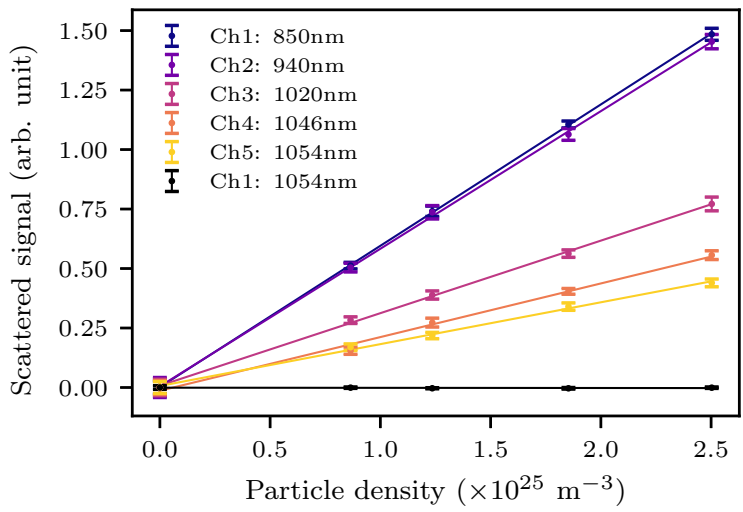

Figure 8. Linear fits of the diode response of a particular channel versus particle density at a given wavelength in air. The slope of the linear fit gives the value of the absolute calibration factor or, when normalized to an arbitrary wavelength, the relative sensitivity of the spectral channel at the wavelengths indicated in the legend. Error bars denote the 2-sigma standard error of the mean.

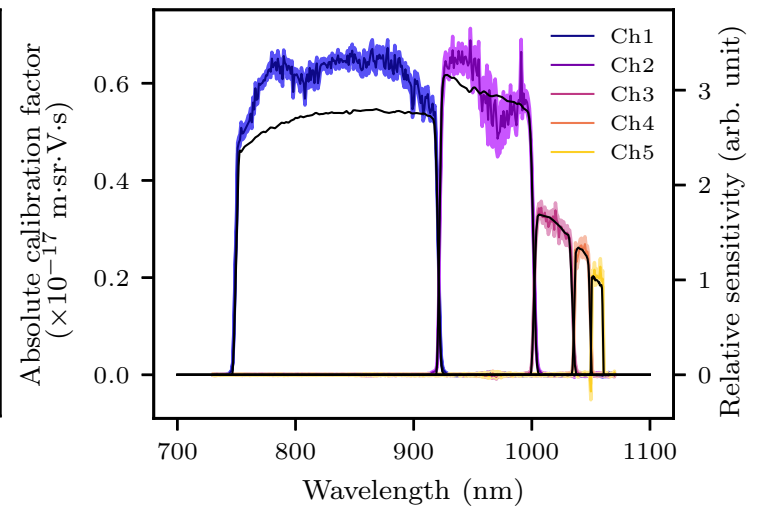

Figure 9. Absolute calibration factor and relative sensitivity normalized to $1054 \mathrm{~nm}$ as determined by Rayleigh scattering in air for all spectral channels. Significant features are visible in the channels, especially near $970 \mathrm{~nm}$. The exact reason is still unknown, but is currently attributed to molecular absorption resulting from the measurements being taken in air. The shaded area represents the 2-sigma standard error of the mean. For comparison, the diffuse-plate spectral calibration is shown in black.

Additionally, the pulse energy would occasionally fail to trigger the PEM energy measurement due to the PEM trigger threshold being set too high. These faults normally occurred around 970 $\mathrm{nm}$, which as seen in figure 6 also corresponds to the lowest output pulse energy. Therefore, fewer data points are available, and the statistical noise increases even further.

\section{Discussion}

Several distinct features present in the Rayleigh scattering calibration clearly set it apart from the diffuse-plate calibration. A strong dip is visible in channel 2 near $970 \mathrm{~nm}$, occurring at a known vibrational mode in water. Additional features in channel 1, including a dip near the edge at 740 $\mathrm{nm}$, also correspond to absorption lines seen in atmospheric measurements [19]. It is therefore 
possible that, because measurements were performed in air, the results are skewed by molecular absorption. It is interesting to note that, as shown in ref. [19], there are not many strong absorption lines in the wavelength range affecting spectral channels 3, 4, and 5, and these channels also show the closest agreement between the two spectral calibration methods.

The data from the argon measurements do not seem to have the same features in channel 1 as seen during measurements in air; however, it is possible that any effect here is masked by increased noise levels, and it is difficult to draw a conclusion based on this comparison. A spike in the data is seen in channel 2 for many polychromators around $970 \mathrm{~nm}$ during argon measurements, but, due to low energy levels, the PEM was not triggered, and it is again difficult to determine the source of error.

The effects of stray light on the measurement are clear, with vacuum measurements in both argon and air using half or more of the detectors' dynamic range. As mentioned briefly in section 3.1, the stray light and Rayleigh scattered signal peaks of many polychromators can be differentiated from one another; all polychromators associated with the M-port observation optics have two distinct peaks, and the core polychromator associated with the N-port observation optics also has a small secondary Rayleigh scattering peak. Though for these polychromators it may be possible to calibrate even with high stray light levels, this peak separation is not visible in half of the system's polychromators, and therefore is not a viable method for calibration of the entire Thomson diagnostic. Additionally, both peaks are still visible in the vacuum case, implying that stray light arrives at the observation optics at nearly the same time as the Rayleigh scattered signal. This also gives a strong indication that there are two significant sources of stray light. It is currently believed that the stray light originates on the entrance and exit vacuum windows, though other effects such as stray light originating from mirror coatings as observed by Nilson et. al. on the DIII-D Thomson system also warrant further investigation [20].

In the current state of the system, there are practical limitations to its implementation. To half the current statistical noise levels, 120 data points are needed. This leads to over an hour of data collection time for a single pressure scan, and a maximum of 5 or 6 pressures in one day. Additionally, for many of the polychromators here, the statistical noise would still not be acceptable, especially for measurements at and below 100 mbar. These considerations exemplify just how critical stray light reduction is for future measurements at W7-X. With sufficient stray light reduction, the aforementioned issues are bypassed. Additionally, through measurements at W7-X are complicated by a maximum pressure differential between the vacuum vessel and cryostat, other fusion devices may not have such limitations, relaxing somewhat the requirements for successful calibrations.

It should also be noted that, while an absolute calibration factor was determined, the values here are an order of magnitude smaller than those obtained via Raman scattering. The Thomson diagnostic at W7-X generally agrees with the line-averaged density determined by dispersion interferometry [21] to within 10 percent, meaning the absolute calibration factor determined via the presented Rayleigh scattering method is not accurate. This is currently attributed to poor beam shaping, a significant coma aberration in the OPO beam profile, and a position mismatch between the OPO and Nd:YAG beams through the vacuum vessel, resulting in the measured pulse energy being unrepresentative of the pulse energy within the scattering volume. 


\subsection{Profile effects}

To further motivate this work, the spectral calibration determined here is applied to a randomly chosen W7-X discharge using two methods, and is compared against a profile generated using the diffuse plate calibration (figures 10 and 11). Note that, because the absolute calibration factor was deemed inaccurate, only the relative spectral calibration determined by Rayleigh scattering is applied; the density profile shown here is determined using a combination of the relative spectral calibration and the absolute calibration factor determined via Raman scattering. The reference wavelength and channel used for determining the relative spectral calibration are $1054 \mathrm{~nm}$ and 5, respectively (see section 3 ).

First, the OPO spectral calibration curves are passed through a median filter for smoothing and applied directly to the profiles ("OPO" in figures 10 and 11). For two polychromators, the high-resolution data was used for channels 4 and 5. This gives an idea of the overall effect of the filter smoothness, the edge positions, and any spectral effects that could be in the system. This is only possible for polychromators in which there is no data loss in any channel, e.g. due to low pulse energy failing to trigger the PEM; therefore not all scattering volumes are available for comparison.

Second, the ratios of the integrals of the diffuse-plate spectral curves are set to equal those of the OPO spectral calibration, effectively scaling the diffuse plate spectral calibration ("Scaled" in figures 10 and 11). This is performed because, as is clearly visible in figure 9, the integral of channel 1 differs between the Rayleigh scattering calibration and the diffuse plate calibration. This also demonstrates the effect of a varying filter channel amplitude while ignoring any potential effects from rough filter curve edges and wavelength offsets between the two spectral calibration methods. This method can be applied even when sections of filter curve data are missing by ignoring areas with missing data during integration.

The resulting temperature and density profiles were calculated using the Minerva framework [22] and are shown in figures 10 and 11, respectively. The profiles are calculated by averaging the data of each scattering volume over 6 seconds. Only volumes that were successfully calibrated are shown, with successful calibration meaning the statistical noise is low enough that the filter curves are clearly visible and gaps in the data due to signal saturation or loss of pulse energy measurements are not present. Only data resulting from one of three lasers is used.

Figure 10 has several interesting features. One, the overall shape of the temperature profile is largely unaffected by the different spectral calibrations, though outliers are less prevalent in the diffuse-plate spectral calibration data. The core profile shape experiences the greatest change; the core temperatures are lowered, resulting in a less pronounced temperature peak. While the points using the direct OPO calibration curves have significantly more scatter, they follow the same trend as the other calibration data.

Differences in the density profile can be seen between the multiple calibration methods in figure 11. The scaled spectral calibration results in a flatter core profile and a similar edge density gradient, though the effects are not particularly pronounced. The direct OPO calibration data suffers from high scatter, likely due to edge effects from low-resolution scans and the OPO linewidth, both of which are being addressed for the next W7-X operational period (sec. 5).

Because the standard error of the sample mean shown in figures 10 and 11 is very low, it is clear that there is a significant change in the profile shapes resulting from the different calibrations. 


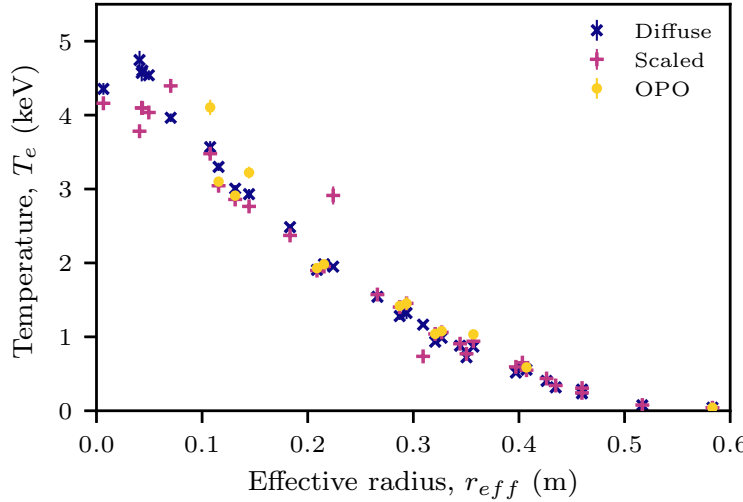

Figure 10. Averaged W7-X temperature profiles from program 20180927.33 from 9.02 to 15.99 seconds (70 pulses) using the diffuse plate (blue crosses), direct OPO (yellow dots), and OPOscaled diffuse plate (purple x's) relative spectral calibrations. Error bars representing the 2-sigma standard error of the sample mean are included, but do not exceed the marker size for most points.

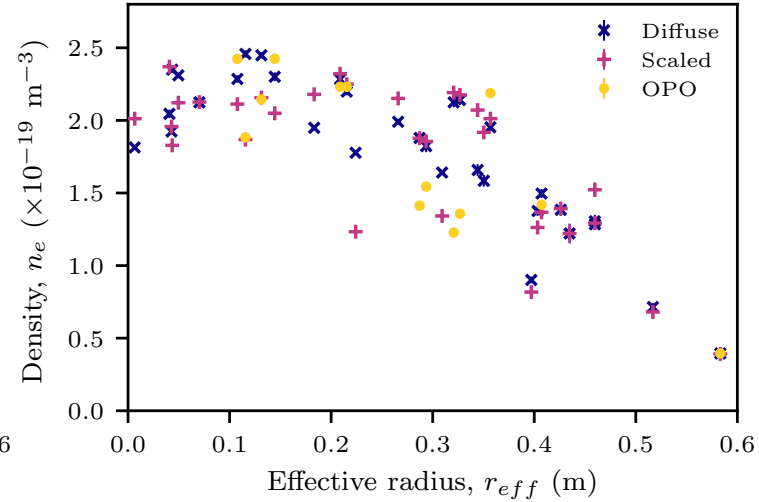

Figure 11. Averaged W7-X density profiles from program 20180927.33 from 9.02 to $15.99 \mathrm{sec}$ onds (70 pulses) using the diffuse plate (blue crosses), direct OPO (yellow dots), and OPOscaled diffuse plate (purple $\mathrm{x}$ 's) relative spectral calibrations in conjunction with the absolute Raman calibration of channel 5. Error bars representing the 2-sigma standard error of the sample mean are included, but do not exceed the marker size for most points.

However, due to the outstanding issues with the spectral calibration presented here, it is not yet possible to determine which spectral calibration method results in the most accurate temperature and density profiles. Several improvements to the current system (see section 5) and ongoing studies of systematic errors in the W7-X Thomson scattering system should make more quantitative comparisons possible for future measurements.

\section{Conclusions and outlook}

Proof-of-principle Rayleigh scattering measurements utilizing an OPO-laser were successfully performed on W7-X, resulting in clear filter curves. Although the absolute calibration factor determined here via Rayleigh scattering differed from that determined by Raman scattering, the relative spectral calibration curves determined via Rayleigh scattering for spectral channels 3, 4, and 5 agree well with those determined via a diffuse-plate scattering method. Differences between the relative spectral calibrations are plainly visible in spectral channels 1 and 2, though the cause of the discrepancy is difficult to determine. This could well result from molecular absorption in air, a faulty mirror coating, or from a more accurate calibration by including the first window and using a similar scattering geometry to the Thomson diagnostic. The small changes observed in the density profile result in the flattening of an otherwise hollow density profile. Although a similar core flattening is seen in the temperature profile of the scaled spectral calibration, the measured temperature profile is largely unaffected by the different calibrations. It is, however, not yet possible to determine the accuracy of one spectral calibration method relative to the other. 
It should be noted that, for the Rayleigh scattering calibration to become an absolute calibration, it is necessary that the pulse energy within a scattering volume is known. This is possible by matching the OPO beam profile and position to the Nd:YAG lasers' profiles and positions at the entrance and exit of the vacuum vessel, leading to an overlap of the OPO and Nd:YAG laser beam paths within each scattering volumes. Cameras focused on the vacuum vessel entrance and exit windows should allow for precise measurement of both the beam position and profile for comparison. The profile matching would additionally have to be confirmed at each wavelength measured.

Currently, it is planned that the Rayleigh calibration system will be installed on W7-X for the next operational period in 2021. Despite the troubles encountered during measurements in argon, figure 3 shows that there is still an appreciable difference between measurements at different pressures, even with a small number of OPO pulses per wavelength and exceedingly high stray light levels. This gives confidence that, with a larger number of pressure steps and a higher number of OPO pulses per wavelength, this calibration technique may be used even with pressures limited to 100 mbar. Beyond this, several improvements are planned to further increase the effectiveness and accuracy of the calibration. First, an additional stray light absorber is to be installed in the laser flight tube of the Thomson scattering diagnostic, reducing the amount of stray light generated during the calibration, allowing for higher laser energy and lower pressures. A laser-seeder is planned for the OPO to further reduce the laser linewidth and achieve higher filter edge resolution. A dedicated set of optics will be designed and installed to allow flexibility in beam shaping to further optimize the system. Finally, a spectrum analyzer will be implemented to measure the laser linewidth and center frequency of each laser pulse.

\section{Acknowledgments}

This work has been carried out within the framework of the EUROfusion Consortium and has received funding from the Euratom research and training programme 2014-2018 and 2019-2020 under grand agreement number 633053. The views and opinions expressed herein do not necessarily reflect those of the European Commission.

\section{References}

[1] T. N. Carlstrom, G. L. Campbell, J. C. DeBoo, R. Evanko, J. Evans, C. M. Greenfield, J. Haskovec, C. L. Hsieh, E. McKee, R. T. Snider, R. Stockdale, P. K. Trost, and M. P. Thomas. Design and operation of the multipulse Thomson scattering diagnostic on DIII-D (invited). Review of Scientific Instruments, 63(10):4901-4906, October 1992.

[2] T. N. Carlstrom, J. H. Foote, D. G. Nilson, and B. W. Rice. Design of the divertor Thomson scattering system on DIII-D. Review of Scientific Instruments, 66(1):493-495, January 1995.

[3] M. J. Walsh, E. R. Arends, P. G. Carolan, M. R. Dunstan, M. J. Forrest, S. K. Nielsen, and R. O'Gorman. Combined visible and infrared Thomson scattering on the MAST experiment. Review of Scientific Instruments, 74(3):1663-1666, March 2003.

[4] Yang Li, Wan Baonian, Zhao Junyu, Hu Qingsheng, Jia Yanqing, Xi Xiaoqi, Han Xiaofeng, and Zang Qing. Design of Thomson Scattering Diagnostic System on EAST. Plasma Science and Technology, 12(3):284-288, June 2010. 
[5] R. Scannell, M. J. Walsh, M. R. Dunstan, J. Figueiredo, G. Naylor, T. O'Gorman, S. Shibaev, K. J. Gibson, and H. Wilson. A 130 point Nd:YAG Thomson scattering diagnostic on MAST. Review of Scientific Instruments, 81(10):10D520, October 2010.

[6] H. Tojo, T. Hatae, T. Sakuma, T. Hamano, K. Itami, Y. Aida, S. Suitoh, and D. Fujie. Design of collection optics and polychromators for a JT-60sa Thomson scattering system. Review of Scientific Instruments, 81(10):10D539, October 2010.

[7] I. Yamada, K. Narihara, H. Funaba, T. Minami, H. Hayashi, T. Kohmoto, and LHD Experiment Group. Recent Progress of the LHD Thomson Scattering System. Fusion Science and Technology, 58(1):345-351, August 2010.

[8] B. Kurzan and H. D. Murmann. Edge and core Thomson scattering systems and their calibration on the ASDEX Upgrade tokamak. Review of Scientific Instruments, 82(10):103501, October 2011.

[9] B P LeBlanc and A Diallo. Alignment of the Thomson scattering diagnostic on NSTX. Journal of Instrumentation, 8(11):C11004, November 2013.

[10] S.A. Bozhenkov, M. Beurskens, A. Dal Molin, G. Fuchert, E. Pasch, M.R. Stoneking, M. Hirsch, U. Höfel, J. Knauer, J. Svensson, H. Trimino Mora, and R.C. Wolf. The Thomson scattering diagnostic at Wendelstein 7-X and its performance in the first operation phase. Journal of Instrumentation, 12(10):P10004, October 2017.

[11] J. Howard, B. W. James, and W. I. B. Smith. Rotational Raman calibration of Thomson scattering. Journal of Physics D: Applied Physics, 12(9):1435, 1979.

[12] B. P. LeBlanc. Thomson scattering density calibration by Rayleigh and rotational Raman scattering on NSTX. Review of Scientific Instruments, 79(10):10E737, October 2008.

[13] D. A. Rasmussen, R. R. Kindsfather, C. E. Thomas, R. P. Gormley, and S. L. Painter. In situ dye laser calibration for Thomson scattering diagnostics. Review of Scientific Instruments, 59(8):1464-1466, August 1988.

[14] S. A. Bozhenkov, S. J. Heym, M. N. A. Beurskens, G. Fuchert, E. Pasch, E. R. Scott, and R. C. Wolf. On using Rayleigh scattering for in situ spectral calibration of Thomson scattering diagnostics. Review of Scientific Instruments, 90(3):033505, March 2019.

[15] Ralph R. Rudder and David R. Bach. Rayleigh scattering of ruby-laser light by neutral gases. JOSA, 58(9):1260-1266, 1968.

[16] Maarten Sneep and Wim Ubachs. Direct measurement of the Rayleigh scattering cross section in various gases. Journal of Quantitative Spectroscopy and Radiative Transfer, 92(3):293-310, May 2005.

[17] Anthony Bucholtz. Rayleigh-scattering calculations for the terrestrial atmosphere. Applied Optics, 34(15):2765, May 1995.

[18] Richard B Miles, Walter R Lempert, and Joseph N Forkey. Laser Rayleigh scattering. Measurement Science and Technology, 12(5):R33, 2001.

[19] ASTM G173-03. Standard Tables for Reference Solar Spectral Irradiances: Direct Normal and Hemispherical on $37^{\circ}$ Tilted Surface. Technical report, ASTM International, West Conshohocken, PA, 2003.

[20] D. G. Nilson, D. N. Hill, J. C. Evans, T. N. Carlstrom, C. L. Hsieh, and R. E. Stockdale. Thomson scattering stray light reduction techniques using a CCD camera. Review of Scientific Instruments, 68(1):704-707, January 1997. 
[21] K.J. Brunner, T. Akiyama, M. Hirsch, J. Knauer, P. Kornejew, B. Kursinski, H. Laqua, J. Meineke, H. Trimiño Mora, and R. C. Wolf. Real-time dispersion interferometry for density feedback in fusion devices. Journal of Instrumentation, 13(09):P09002, September 2018.

[22] J Svensson and A Werner. Large Scale Bayesian Data Analysis for Nuclear Fusion Experiments. In 2007 IEEE International Symposium on Intelligent Signal Processing, pages 1-6, Alcala de Henares, Spain, 2007. IEEE. 